\title{
A Simple and Efficient Method for Synthesizing Te Nanowires from CdTe Nanoparticles with EDTA as Shape Controller under Hydrothermal Condition
}

\author{
Fangfang Xue, Ning Bi, Jiangong Liang, and Heyou Han \\ College of Science, State Key Laboratory of Agricultural Microbiology, Huazhong Agricultural University, Wuhan 430070, China \\ Correspondence should be addressed to Jiangong Liang, liangjg@mail.hzau.edu.cn
}

Received 11 March 2012; Accepted 22 May 2012

Academic Editor: Jianguang Jia

Copyright (c) 2012 Fangfang Xue et al. This is an open access article distributed under the Creative Commons Attribution License, which permits unrestricted use, distribution, and reproduction in any medium, provided the original work is properly cited.

\begin{abstract}
We developed a simple and efficient method for synthesizing Te nanowires from CdTe nanoparticles with ethylenediaminetetraacetic acid disodium salt dehydrate (EDTA) as shape controller under hydrothermal condition. The system could both complete the transformation to Te and reduce the interference of CdTeS by adjusting the concentration of EDTA, which was proved by inductively coupled plasma mass spectrometry, X-ray diffraction patterns, and Raman spectra. It was found that the as-prepared Te nanowires display strong fluorescence emission in the blue-violet region. The nanowires exhibit a pretty good morphology with the average diameter of ca. $30 \mathrm{~nm}$ and a length up to micrometer scale. Moreover, a possible transformation mechanism of CdTe nanoparticles into Te nanowires is also discussed.
\end{abstract}

\section{Introduction}

One-dimensional (1D) semiconductor nanostructures, such as nanowires, rods, tubes, and belts, have been a significant aspect of today's endeavours in nanotechnology, due to their special optical, magnetic, electrical, and chemical properties [1-4]. Up to now, many techniques have been designed to synthesize one-dimensional semiconductor nanostructures, such as electrochemical synthesis [5], microwave-assisted synthesis $[6,7]$, template method [8], precursor [9], selfseeding solution process [10], polyol process [11], spontaneous route $[12,13]$, and solution-based chemical process $[14,15]$.

It is very important to control the formation and morphology of semiconductor nanostructure in the exploitation of novel properties [13, 15-17]. Progress has been made in synthesizing $1 \mathrm{D}$ nanostructures using semiconductor nanoparticles [18]. According to a pioneer study conducted in 2002 [3], CdTe nanoparticles could spontaneously reorganize into nanowires after controlled removal of the protective shell of organic stabilizer and storage in darkness at room temperature under ambient conditions for a continuous growth of 7 days. In 2005, to synthesize Se and Te nanowires and investigate their possible transition mechanism, the researchers used stabilizer-depleted CdTe and CdSe nanoparticles and introduced ethylenediaminetetraacetic acid disodium salt dihydrate (EDTA) to the nanoparticles [12]. In 2006, they continued to synthesize spontaneously angled Te nanocrystals such as checkmarks, X-marks, and other unusual shapes and confirmed the transition mechanism of the system $[19,20]$. However, it is necessary to develop a simpler and more efficient method for synthesizing nanowires.

In this study, hydrothermal method was used to synthesize Te nanowire in a hexagonal structure from CdTe nanoparticles with EDTA as shape controller. The effect of EDTA on the chemical transformation and morphology control of the nanowires was explored in details. This technique of nanowire synthesis is safe, and meanwhile the quality and the optical activities of the nanowires are very significant. This method, therefore, will find great potential applications in the processes of chemical transformation and synthesizing 1D nanostructures using semiconductor nanoparticles. In particular, this crystalline Te nanowires display strong fluorescence $(\mathrm{FL})$ emission in the blue-violet region, which is of great importance for optoelectronic applications [21]. 


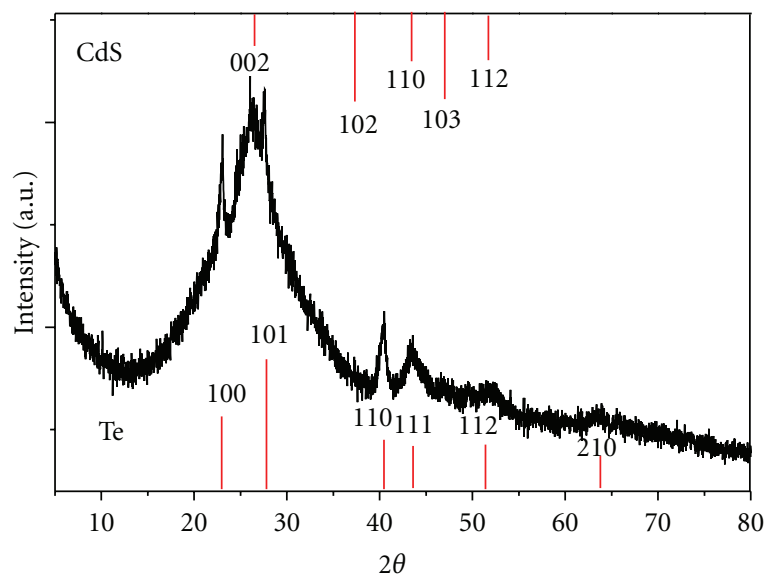

(A)

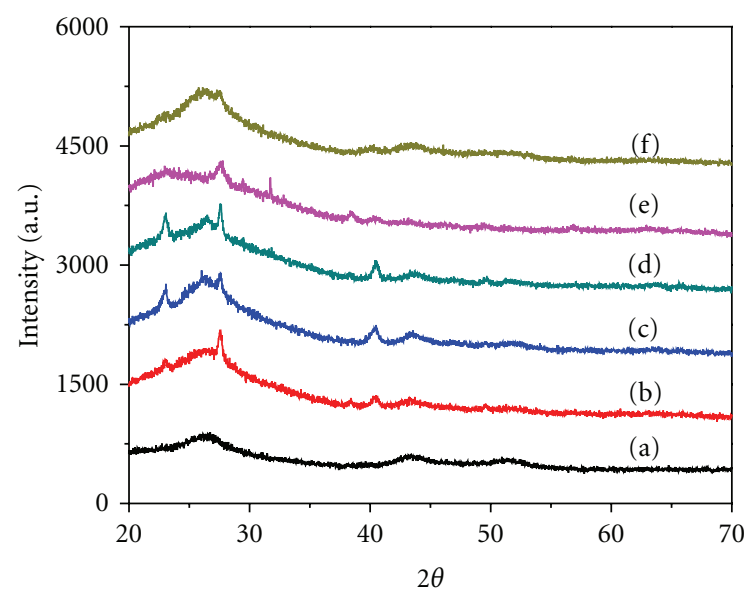

(B)

FIGURE 1: (A) XRD patterns of the as-synthesized nanowires with $0.10 \mathrm{~g} \cdot \mathrm{L}^{-1}$ and (B): (a) $0 \mathrm{~g} \cdot \mathrm{L}^{-1} \mathrm{EDTA}$; (b) $0.050 \mathrm{~g} \cdot \mathrm{L}^{-1} \mathrm{EDTA}$; (c) 0.10 $\mathrm{g} \cdot \mathrm{L}^{-1}$ EDTA; (d) $0.50 \mathrm{~g} \cdot \mathrm{L}^{-1} \mathrm{EDTA}$; (e) $1.0 \mathrm{~g} \cdot \mathrm{L}^{-1} \mathrm{EDTA}$; (f) $6.0 \mathrm{~g} \cdot \mathrm{L}^{-1}$ EDTA.

\section{Experimental Methods}

NaHTe was prepared according to the previously published procedure with several modifications [22, 23]. Briefly, a mixture of $0.0107 \mathrm{~g}$ of tellurium ( $\mathrm{Te}$ ) powder and $0.0354 \mathrm{~g}$ of sodium borohydride (molar ratio of Te to $\mathrm{NaBH}_{4}$ is $1: 3$ ) was first loaded into a $10 \mathrm{~mL}$ bottle, then $0.2 \mathrm{~mL}$ of $\mathrm{N}_{2}$ saturated ultrapure water was added into the bottle to react at $4^{\circ} \mathrm{C}$ until the black Te powder fully disappeared and white sodium tetraborate precipitation appeared at the bottom of the bottle. The as-prepared NaHTe solution was used as Te precursor in the following reaction.

$0.0357 \mathrm{~g} \mathrm{CdCl}_{2}$ and $100 \mathrm{~mL}$ ultrapure water were transferred to a three-necked flask with a condenser attached and kept bubbling with highly pure $\mathrm{N}_{2}$ for 20 mins. This solution was then mixed with $15 \mu \mathrm{L}$ thioglycolic acid (TGA) and $0.0227 \mathrm{~g}$ L-cysteine (L-cys). After that, $1.0 \mathrm{~mol} \cdot \mathrm{L}^{-1} \mathrm{NaOH}$ was added to adjust its $\mathrm{pH}$ to 11 when the mixture became clear. $2.0 \mathrm{~mL}$ freshly prepared NaHTe solution was rapidly injected into the solution under vigorous stirring. The obtained solution was heated at $100^{\circ} \mathrm{C}$ for $3.5 \mathrm{~h}$, and then was transferred to autoclave at $150^{\circ} \mathrm{C}$ for $1 \mathrm{~h}$ with a certain concentration of EDTA solution at $\mathrm{pH}$ 9. During the process, the colour turned from orange to deep blue, the typical colour as found previously for Te nanowires synthesized by the hydrothermal method [6]. The as-prepared product was centrifuged with acetone and then stored in a Medical refrigerator at $4^{\circ} \mathrm{C}$ for future use.

Fluorescence measurements were performed in a $3.0 \mathrm{~mL}$ quartz cell with an optical pathlength of $1.0 \mathrm{~cm}$ at room temperature using a RF-5301 Luminescence Spectrometer (SHIMADZU, Japan), with the scanning speed set at fast. $\mathrm{X}$-ray diffraction (XRD) patterns were taken on a Rigaku $\mathrm{D} / \mathrm{MAX}-\mathrm{RB}$ diffractometer with $\mathrm{Cu} \mathrm{K}_{\alpha}$ radiation $(\lambda=$ $0.15406 \mathrm{~nm}$ ). The transmission electron microscopy (TEM) images were performed using H-7650 (Hitachi, Japan) with an accelerating voltage of $200 \mathrm{kV}$. The scanning electron microscopy (SEM) images of catalysts were obtained by a
Philips XL30 FEG scanning electron microscope. The Raman spectra were acquired with an inVia micro-Raman spectra spectroscopy system (Renishow, UK) in a spectral range of $100-2000 \mathrm{~cm}^{-1}$, equipped with a $\mathrm{He}-\mathrm{Ne}$ laser excitation source emitting wavelength at $633 \mathrm{~nm}$. X-ray photoelectron spectra (XPS) were recorded on a VG multilab 2000 X-ray photoelectron spectrometer using $\mathrm{Al} \mathrm{K}_{\mathrm{a}}$ radiation $(h \nu=$ $1486.58 \mathrm{eV}$ ) as the exciting source. The inductively coupled plasma mass spectrometry (ICP-MS) instrument used was an Aglient $7500 \mathrm{ce}$. All pH measurements were tested with a Model MP 120 pH meter (Mettler-Toledo Instruments Ltd., Swiss).

\section{Results and Discussion}

Panels (A) and (B) of Figure 1 show the XRD patterns of the freshly prepared product with different concentrations of EDTA, which proved that transformation of CdTe-CdS/Te exists and CdTeS could be effectively regulated just by the introduction of EDTA. As shown in Figure 1(A), the spectrum of the sample was in accordance with the values in the standard card of hexagonal Te (JCPDS no. 36-1452) [6], with the major reflections being at 100, 101, 110, 111, 112 , and 210, respectively. These peaks can be indexed to perfect hexagonal structure of trigonal Te with the cell constants of $a=4.4579 \AA$ and $c=5.927 \AA$. Strong Te peaks undoubtedly confirmed that hexagonal Te really exists in the product. The four reflections at 002, 110, 103 and 112 are clear and support hexagonal crystal structure of CdS [24]. The reflections at 102 are poor due to the overlapping of peaks. These peaks in this pattern can be indexed to the hexagonal phase of CdS with the cell constants $a=4.14092 \AA$ and $c=6.7198 \AA$, which are in good agreement with the standard literature data (JCPDS no. 77-2306). As previously reported [19], the transformation products were composed of anisotropic Te and CdTeS component after EDTA were added into the crude CdTe nanoparticle (NP) solution without the step of stabilizer depletion. However, because 
the ligands bond to the surface atoms were dynamically "on and off" [25], hydrothermal technology was adopted in our study to accelerate the dynamic process. CdTeS without centrifugation in methanol or 2-propanol to deplete the stabilizer shell. The image of Figure 1(b) confirmed the process, which shows that the peaks of CdS gradually disappeared with the increasing EDTA concentration.

The chemical composition of as-prepared 1D nanowires were measured by ICP-MS, which is considered as a powerful technique for trace and ultra-trace element analysis among research fields [26]. Before the ICP-MS measurements, the as-prepared samples were first purified and then dissolved by aqua regia and diluted by ultrapure water. Table 1 displays the relationship between EDTA concentrations and the element content of the nanowires. Along with the increase of the EDTA concentration, the maximum content of $\mathrm{Te}$ in nanowires emerged at $0.50 \mathrm{~g} \cdot \mathrm{L}^{-1}$ and then showed a significant decrease, indicating that the components of the nanowires could be effectively accommodated by adjusting the concentration of EDTA.

XPS was also used to characterize the surface chemical composition of the as-prepared nanowires. Shown in Figure 2 are the XPS spectra of the obtained nanowires without EDTA (Figure 2(a)) and with $0.50 \mathrm{~g} \cdot \mathrm{L}^{-1}$ EDTA (Figure 2(b)). The measured $\mathrm{Cd} / \mathrm{Te} / \mathrm{S}$ atomic ratio of $1.00: 22.9: 9.53$ without EDTA and $1.91: 56.3: 1.00$ with $0.50 \mathrm{~g} \cdot \mathrm{L}^{-1}$ EDTA indirectly reflect the surface composition of the $1 \mathrm{D}$ nanowires. The peak of $S$ could also be detected from the sample with $0.50 \mathrm{~g} \cdot \mathrm{L}^{-1}$ EDTA due to formation of CdTeS. The S atoms can only come from the thiols of stabilizer, and the addition of EDTA was supposed to have partially removed the stabilizers from the nanowires to decrease the thiols in the surface.

The influence of the EDTA concentrations on the shape of the Te nanowires was studied by TEM and SEM. As shown in Figures 3-4, the products are exclusively anisotropic. To observe all the forms of the nanowires present, the nanowires showed noticeable variance while increasing the concentration of EDTA. From the images, it can be seen that, without EDTA, the nanowires are not very uniform and particularly the length and the quantity of the product are very limited. By introducing EDTA, both the morphology and the quantity of the nanowires are improved effectively. When the concentration of EDTA was $0.10 \mathrm{~g} \cdot \mathrm{L}^{-1}$, the uniform and long nanowires were obtained. The images in Figure 3(c) and Figure 4(c) show the long nanowires with high aspect ratios, an average diameter of $30 \mathrm{~nm}$, and lengths up to micrometer scale. When the concentration of EDTA was lower than $0.10 \mathrm{~g} \cdot \mathrm{L}^{-1}$, the morphology tends to become gradually uniform, suggesting that the addition of EDTA improves the transformation of nanoparticles to nanowires, and hence the quantity of nanowires was remarkably enhanced. When the concentration of EDTA was more than $0.10 \mathrm{~g} \cdot \mathrm{L}^{-1}$, an agglomeration occurred and the obtained nanowires became shorter. In addition, the images clearly demonstrate that the resulting nanowires possess good monodispersity in solution. The dispersion of $\mathrm{Te}$ nanowires in water was deep blue, which is the typical colour as found previously for Te nanowires $[6,27]$. Meanwhile, the flexibility of the material was shown to be pretty good. EDTA,
TABLE 1: The effect of EDTA concentrations on the element content ratio of Te to Cd in nanowires by ICP-MS.

\begin{tabular}{lcccccc}
\hline $\begin{array}{l}\text { Concentration }\left(\mathrm{g} \cdot \mathrm{L}^{-1}\right) \text { of } \\
\text { EDTA }\end{array}$ & 0 & 0.050 & 0.10 & 0.50 & 1.0 & 6.0 \\
$\begin{array}{l}\text { The content ratio of Te to } \\
\text { Cd }\end{array}$ & 0.807 & 3.321 & 7.685 & 88.78 & 7.752 & 0.790 \\
\hline
\end{tabular}

therefore, could have played a crucial role in the formation and growth of the Te nanowires.

Figure 5 depicts the Raman scattering spectra of asprepared nanowires with different concentrations of EDTA. The characteristic vibration peaks at 121.4, 140.8, and $260.7 \mathrm{~cm}^{-1}$ of Te are close to those reported previously, but have shown an obvious shift to high frequency $[28,29]$. The $121.4 \mathrm{~cm}^{-1}$ and $140.8 \mathrm{~cm}^{-1}$ wavelength bands were assigned to $A_{1}$ and $E$ phonon vibration modes of tellurium [30]. As depicted in Figure 5 and the inset figure, in the sample without EDTA, the wavelength number of $139.3 \mathrm{~cm}^{-1}$ and $157.7 \mathrm{~cm}^{-1}$ was attributed to longitudinal optical (LO) mode and weak transverse optical (TO) mode in CdTe, while the $269.8 \mathrm{~cm}^{-1}$ and $291.4 \mathrm{~cm}^{-1}$ wavelength bands were related to the CdS phonon vibrational mode. Under hydrothermal conditions, when the thiol compound was hydrolyzed in alkaline solution, CdTeS formed on the surface of original nanowires, which has proved that CdTeS shell actually envelops the nanowires [31]. However, the corresponding peaks in the spectra with EDTA are not so distinct maybe due to the limition of the content of CdTeS shell. Therefore, all this also has confirmed that only with the help of EDTA can the hydrothermal method regulate the components of CdTeS effectively.

Figure 6 shows the FL emission spectra of nanowires with different concentrations of EDTA. The FL emission spectra excited at $230 \mathrm{~nm}$ and measured at room temperature with the nanowires sample dispersed in ultrapure water. The spectra showed two obvious emission peaks: a broad weak peak at around $300 \mathrm{~nm}$ due to excitonic emission and a narrow sharp peak at $370 \mathrm{~nm}$ due to carrier recombination caused by surface trap states of the nanowires. Different from what was reported previously [27], a narrow sharp peak was detected at $370 \mathrm{~nm}$ due to carrier recombination caused by surface trap states of the nanowires. The introduction of the EDTA was assumed to have led to a significant increase in the amount of excitonic emission peak and surface trap state emission. It is well known that the trap state emission relates to the surface chemistry and the structure of the nanowire [32]. Therefore, we infer that the introduction of EDTA results in certain changes in the surface state. The maximum FL intensity of trap state emission was found to emerge at the concentration of $0.50 \mathrm{~g} \cdot \mathrm{L}^{-1}$ (Figure $6(\mathrm{~d})$ ), which is consistent with the result of ICP-MS. The strong optical properties in the blue-violet region of the Te nanowires observed suggest that they may have potential applications in optoelectronic scales. 

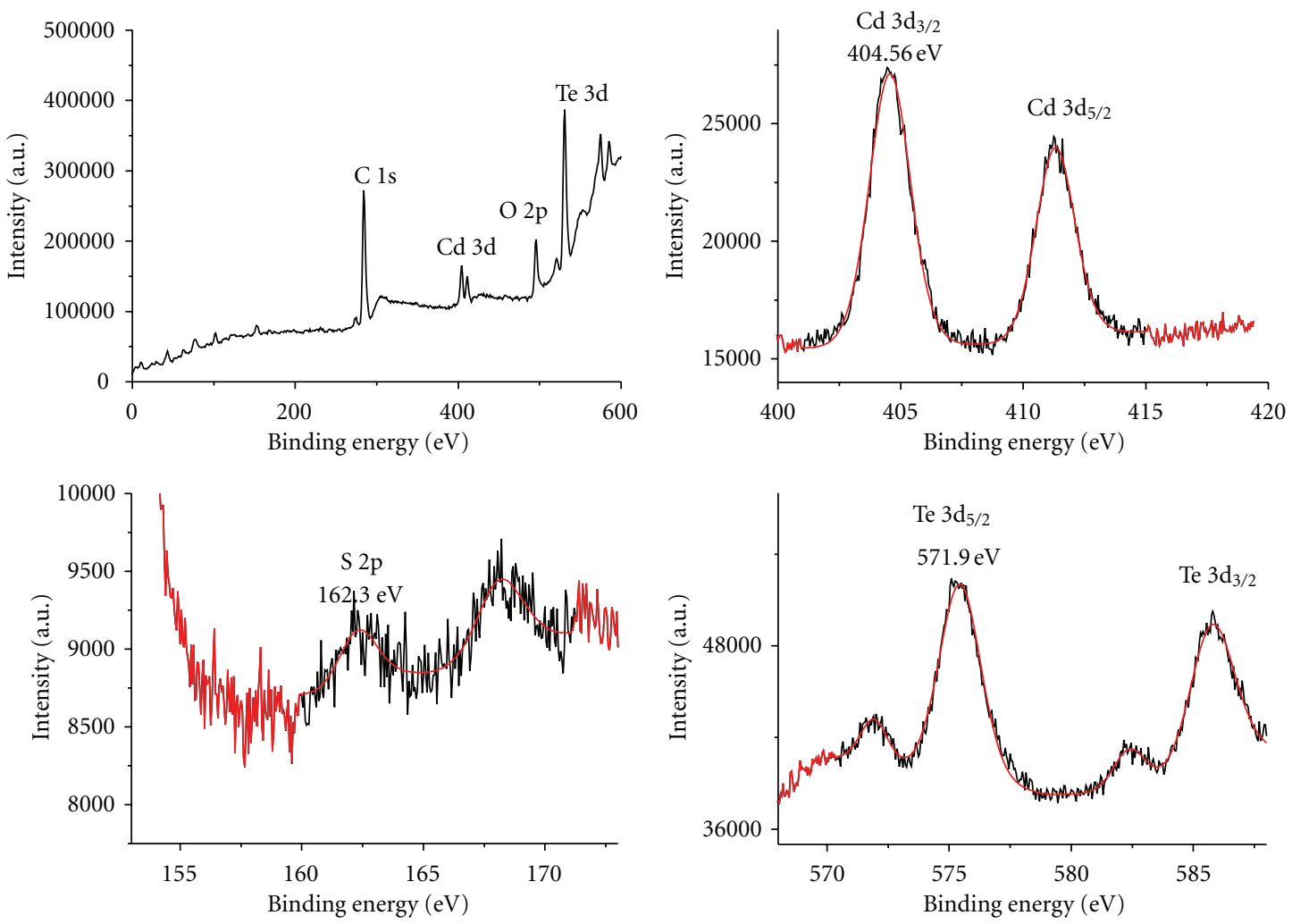

(a)
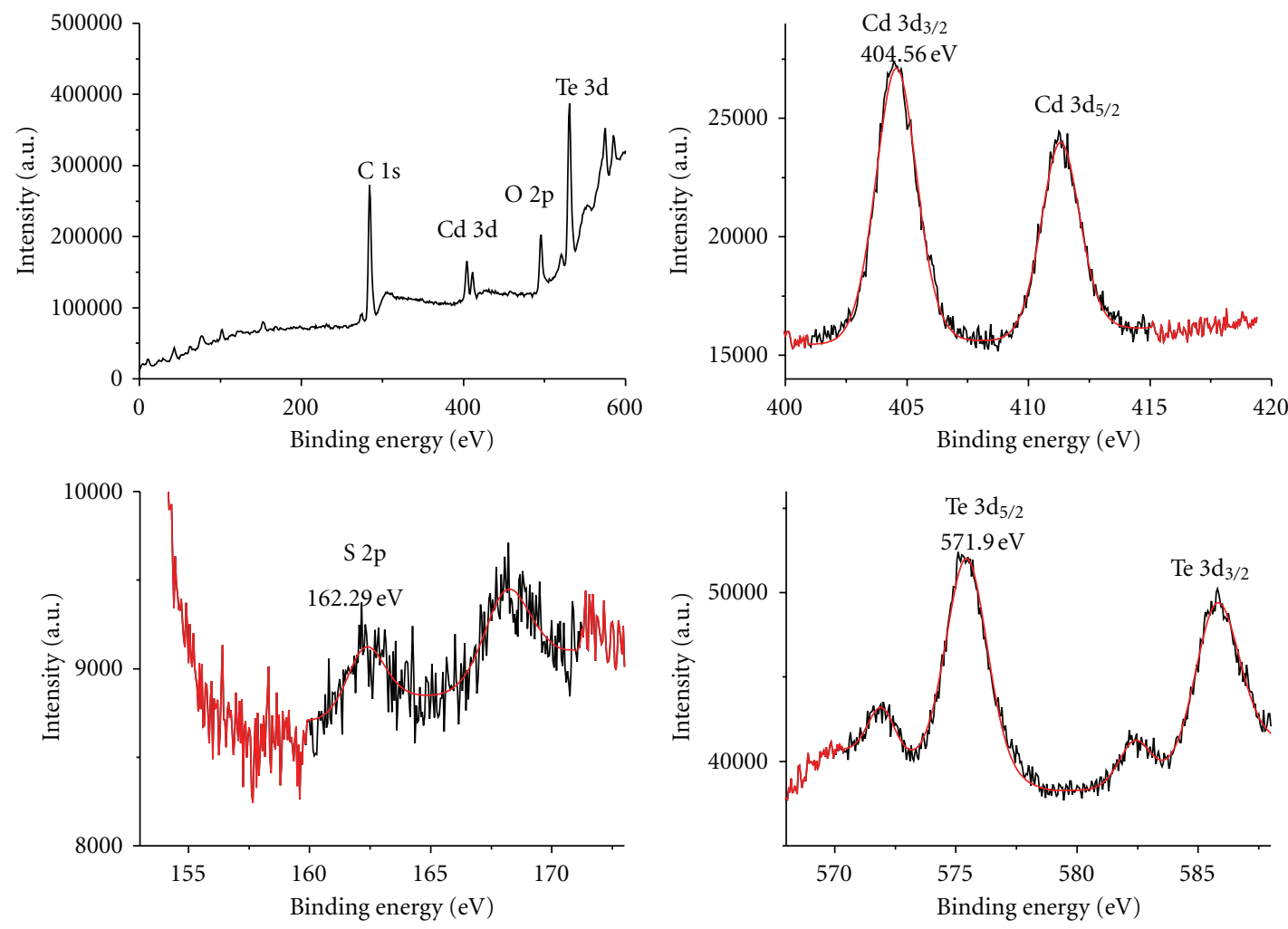

(b)

FIGURE 2: XPS spectra of the obtained nanowires (a) without EDTA and (b) with $0.50 \mathrm{~g} \cdot \mathrm{L}^{-1}$ EDTA. 


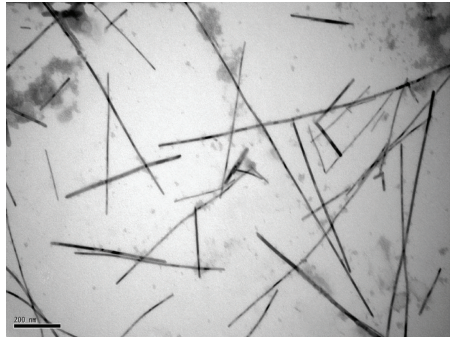

(a)

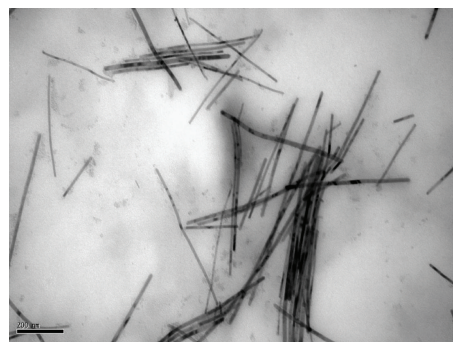

(d)

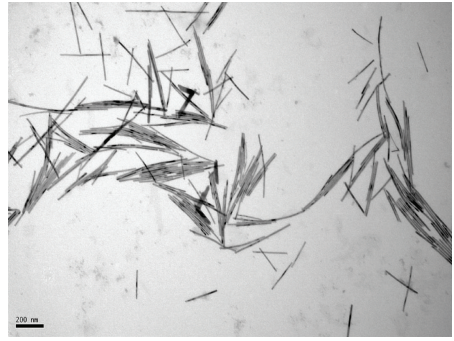

(b)

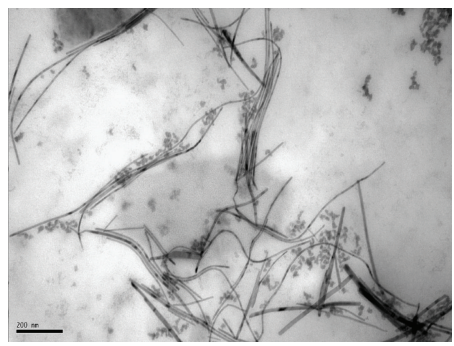

(e)

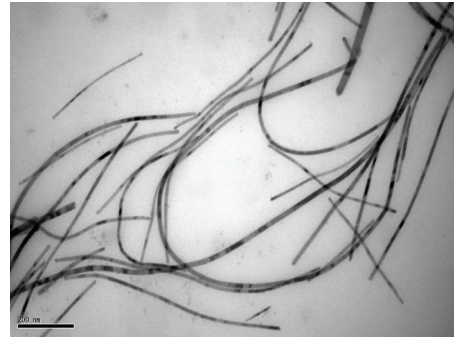

(c)

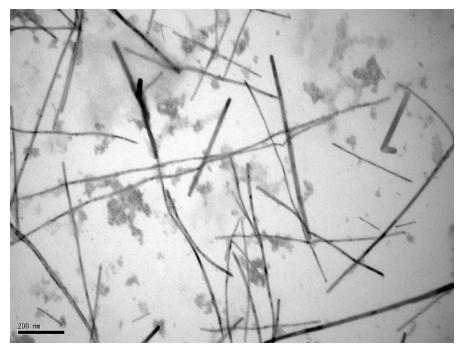

(f)

Figure 3: TEM images of nanowires with (a) $0 \mathrm{~g} \cdot \mathrm{L}^{-1}$, (b) $0.050 \mathrm{~g} \cdot \mathrm{L}^{-1}$, (c) $0.10 \mathrm{~g} \cdot \mathrm{L}^{-1}$, (d) $0.50 \mathrm{~g} \cdot \mathrm{L}^{-1}$, (e) $1.0 \mathrm{~g} \cdot \mathrm{L}^{-1}$, and (f) $6.0 \mathrm{~g} \cdot \mathrm{L}^{-1} \mathrm{EDTA}$. The scale bar is $200 \mathrm{~nm}$.

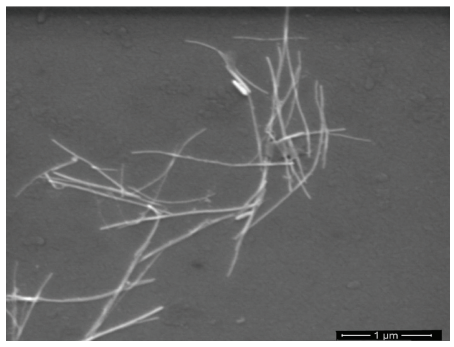

(a)

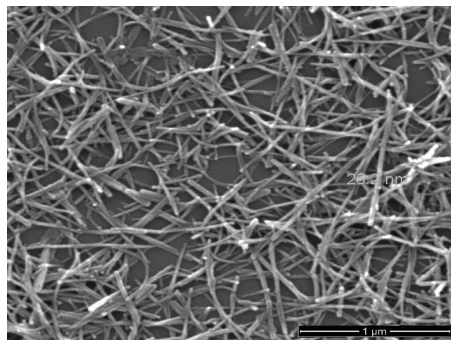

(d)

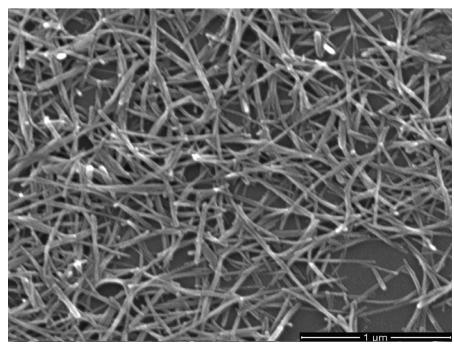

(b)

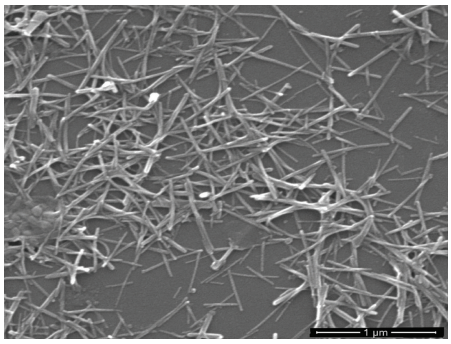

(e)

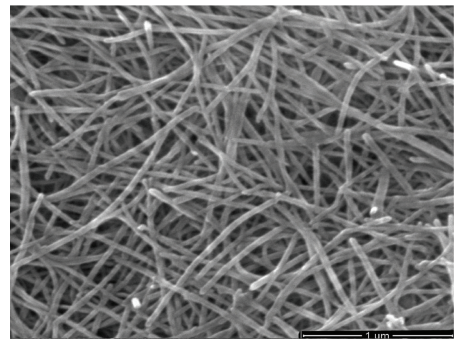

(c)

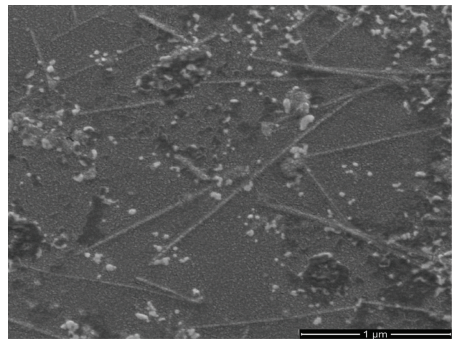

(f)

FIGURE 4: SEM images of nanowires with (a) $0 \mathrm{~g} \cdot \mathrm{L}^{-1}$, (b) $0.050 \mathrm{~g} \cdot \mathrm{L}^{-1}$, (c) $0.10 \mathrm{~g} \cdot \mathrm{L}^{-1}$, (d) $0.50 \mathrm{~g} \cdot \mathrm{L}^{-1}$, (e) $1.0 \mathrm{~g} \cdot \mathrm{L}^{-1}$, and (f) $6.0 \mathrm{~g} \cdot \mathrm{L}^{-1} \mathrm{EDTA}$. The scale bar is $1.0 \mu \mathrm{m}$.

A possible mechanism by which CdTe nanoparticles are transformed into Te nanowires is suggested as follows [12, 33-36]:

$$
\begin{gathered}
\mathrm{CdTe}_{(\mathrm{NP})}+\mathrm{EDTA} \leftrightharpoons \mathrm{CdTe}_{(\mathrm{NP})} \cdot \mathrm{CdTe}(\mathrm{EDTA})^{2-}+\mathrm{Te}^{2-} \\
2 \mathrm{Te}^{2-}+\mathrm{O}_{2}+2 \mathrm{H}_{2} \mathrm{O} \longrightarrow 2 \mathrm{Te}+4 \mathrm{OH}^{-} \\
\mathrm{Te}+2 \mathrm{HSR}+2 \mathrm{OH}^{-} \longrightarrow \mathrm{Te}+2 \mathrm{R}+2 \mathrm{~S}^{2-}+2 \mathrm{H}_{2} \mathrm{O} \\
\mathrm{CdTe}_{(\mathrm{NP})} \cdot \mathrm{CdTe}(\mathrm{EDTA})^{2-}+\mathrm{S}^{2-} \leftrightharpoons \mathrm{CdTeS}_{(\mathrm{NP})}+\mathrm{EDTA}^{4-}
\end{gathered}
$$

The as-prepared CdTe nanoparticles stabilized with Lcys and TGA were redispersed into a certain concentration of EDTA solution at $\mathrm{pH} 9$ (1). In the presence of EDTA, inherent instability of $\mathrm{Te}^{2-}$ and $\mathrm{HTe}^{-}$in the solution can be gradually released in water, due to their strong reducing character of being easily oxidized by dissolved oxygen and finally transformed to Te (2) [33]. Due to its relatively low viscosity, water was a good medium for tellurium atoms to diffuse through, and the tellurium atoms were solvated in the solution phase. Meanwhile, because an excessive amount of L-cys and TGA stabilizers were involved in the transformation reaction [19], the modified reagents containing thiol 


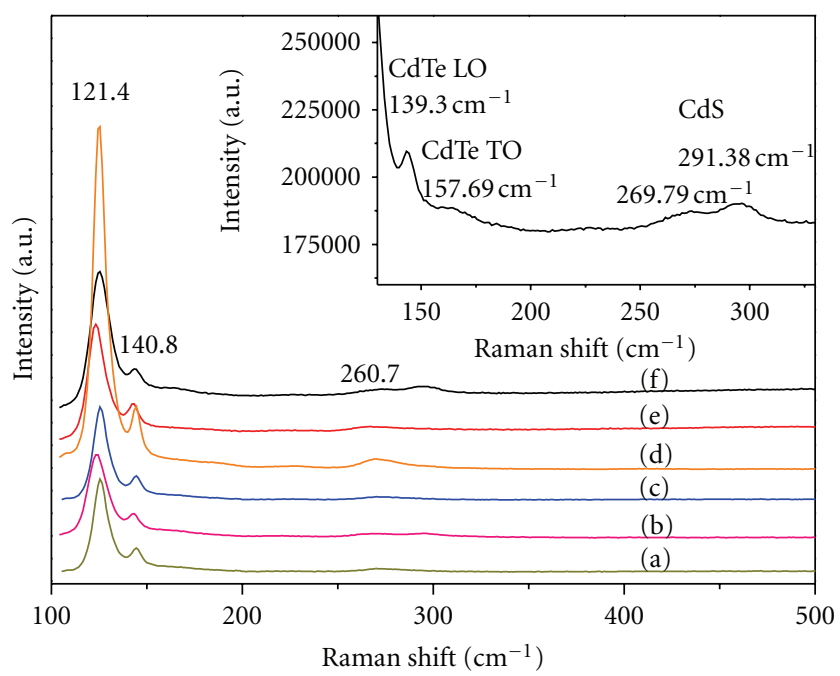

FIGURE 5: Raman scattering spectra of nanowires with (a) $0 \mathrm{~g} \cdot \mathrm{L}^{-1}$, (b) $0.050 \mathrm{~g} \cdot \mathrm{L}^{-1}$, (c) $0.10 \mathrm{~g} \cdot \mathrm{L}^{-1}$, (d) $0.50 \mathrm{~g} \cdot \mathrm{L}^{-1}$, (e) $1.0 \mathrm{~g} \cdot \mathrm{L}^{-1}$ and (f) $6.0 \mathrm{~g} \cdot \mathrm{L}^{-1}$ EDTA. (Inset: the Raman spectrum of as-prepared Te nanowires with the concentration of $0.50 \mathrm{~g} \cdot \mathrm{L}^{-1}$ EDTA.)

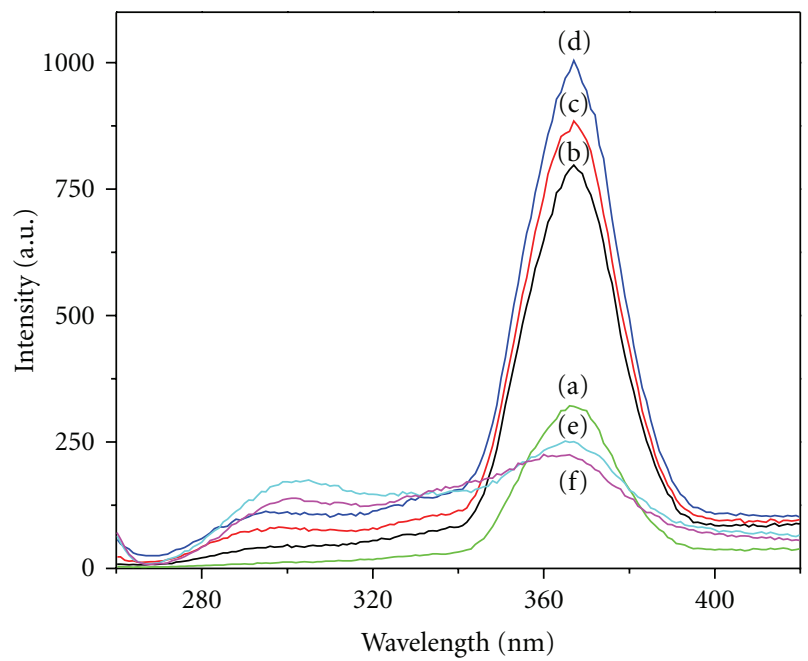

FIGURE 6: FL emission spectra of nanowires with (a) $0 \mathrm{~g} \cdot \mathrm{L}^{-1}$, (b) $0.050 \mathrm{~g} \cdot \mathrm{L}^{-1}$, (c) $0.10 \mathrm{~g} \cdot \mathrm{L}^{-1}$, (d) $0.50 \mathrm{~g} \cdot \mathrm{L}^{-1}$, (e) $1.0 \mathrm{~g} \cdot \mathrm{L}^{-1}$, and (f) 6.0 $\mathrm{g} \cdot \mathrm{L}^{-1}$ EDTA.

reacted with the tellurium atoms, and then released $S^{2-}(3)$. Finally, $\mathrm{S}^{2-}$ interacted with the complex to form CdTeS (4), which was proved by XRD results.

What's more, the manipulation of EDTA concentrations can effectively regulate the amount of Te, and the introduction of the hydrothermal route increases the reaction temperature, which in turn results in the acceleration of the dynamic process of the ligands to the surface atoms. Besides, the long- and short-range of interparticle forces lead to the formation, and the mixture of different ligands contributing to the anisotropic growth of nanowires [34-37].

\section{Conclusions}

In conclusion, we have reported the nonspontaneous synthesis of Te nanowires by using the partially destabilized hydrothermal method and adjusting the concentration of EDTA. The resulting nanowires exhibit both an excellent uniformity in diameter and a high aspect ratio. The adjustment of the concentration of EDTA offers a convenient means of controlling the morphology of nanowires. The strong optical properties in the blue-violet region may have potential applications in optoelectronic scales labels.

\section{Acknowledgments}

The authors gratefully acknowledge the support for this research by National Nature Science Foundation of China (20905028 and 20975042), the Fundamental Research Funds for the Central Universities (2011PY009), and Huazhong Agricultural University Scientific \& Technological Selfinnovation Foundation (2009SC021). They also thank Mr. Hanchang Zhu for his revision of the language of this paper.

\section{References}

[1] M. I. B. Utama, J. Zhang, R. Chen et al., "Synthesis and optical properties of II-VI 1D nanostructures," Nanoscale, vol. 4, pp. 1422-1435, 2012.

[2] H. Qi, O. J. Glembocki, and S. M. Prokes, "Plasmonic properties of vertically aligned nanowire arrays," Journal of Nanomaterial, vol. 2012, Article ID 843402, 7 pages, 2012.

[3] Z. Tang, N. A. Kotov, and M. Giersig, "Spontaneous organization of single CdTe nanoparticles into luminescent nanowires," Science, vol. 297, no. 5579, pp. 237-240, 2002.

[4] Y. S. Zhao, H. Fu, F. Hu, A. Peng, W. Yang, and J. Yao, “Tunable emission from binary organic one-dimensional nanomaterials: an alternative approach to white-light emission," Advanced Materials, vol. 20, no. 1, pp. 79-83, 2008.

[5] X. J. Wu, F. Zhu, C. Mu et al., "Electrochemical synthesis and applications of oriented and hierarchically quasi-1D semiconducting nanostructures," Coordination Chemistry Reviews, vol. 254, no. 9-10, pp. 1135-1150, 2010.

[6] J. W. Liu, F. Chen, M. Zhang, H. Qi, C. L. Zhang, and S. H. Yu, "Rapid microwave-assisted synthesis of uniform ultralong te nanowires, optical property, and chemical stability," Langmuir, vol. 26, no. 13, pp. 11372-11377, 2010.

[7] Q. Lu, F. Gao, and S. Komarneni, "Microwave-assisted synthesis of one-dimensional nanostructures," Journal of Materials Research, vol. 19, no. 6, pp. 1649-1655, 2004.

[8] G. Cao and D. Liu, "Template-based synthesis of nanorod, nanowire, and nanotube arrays," Advances in Colloid and Interface Science, vol. 136, no. 1-2, pp. 45-64, 2008.

[9] J. Ma, J. Lian, X. Duan et al., "Growth of tellurium nanowire bundles from an ionic liquid precursor," CrystEngComm, vol. 13, no. 7, pp. 2774-2778, 2011.

[10] B. Mayers and Y. Xia, "One-dimensional nanostructures of trigonal tellurium with various morphologies can be synthesized using a solution-phase approach," Journal of Materials Chemistry, vol. 12, no. 6, pp. 1875-1881, 2002.

[11] B. Mayers and Y. N. Xia, "Formation of tellurium nanotubes through concentration depletion at the surfaces of seeds," Advanced Materials, vol. 14, pp. 279-282, 2002.

[12] Z. Tang, Y. Wang, K. Sun, and N. A. Kotov, "Spontaneous transformation of stabilizer-depleted binary semiconductor nanoparticles into selenium and tellurium nanowires," Advanced Materials, vol. 17, no. 3, pp. 358-363, 2005. 
[13] J. Yang, Y. Gao, J. W. Kim et al., "Self-reorganization of CdTe nanoparticles into two-dimensional $\mathrm{Bi}_{2} \mathrm{Te}_{3} / \mathrm{CdTe}$ nanosheets and their thermoelectrical properties," Physical Chemistry Chemical Physics, vol. 12, no. 38, pp. 11900-11904, 2010.

[14] Y. Zhu, T. Mei, Y. Wang, and Y. Qian, "Formation and morphology control of nanoparticles via solution routes in an autoclave," Journal of Materials Chemistry, vol. 21, no. 31, pp. 11457-11463, 2011.

[15] H. Y. Zhu, Y. Lan, X. P. Gao et al., "Phase transition between nanostructures of titanate and titanium dioxides via simple wet-chemical reactions," Journal of the American Chemical Society, vol. 127, no. 18, pp. 6730-6736, 2005.

[16] Z. Tang, P. Podsiadlo, B. S. Shim, J. Lee, and N. A. Kotov, "The effect of stabilizer density on transformation of CdTe nanoparticles induced by Ag cations," Advanced Functional Materials, vol. 18, no. 23, pp. 3801-3808, 2008.

[17] J. Yang, Y. Zhou, S. Zheng et al., "Self-reorganization of CDTE nanoparticles into near-infrared $\mathrm{Hg}_{1-\mathrm{x}} \mathrm{Cd}_{\mathrm{x}} \mathrm{Te}$ nanowire networks," Chemistry of Materials, vol. 21, no. 14, pp. 3177-3182, 2009.

[18] H. Zhang, D. Wang, and H. Möhwald, "Ligand-selective aqueous synthesis of one-dimensional CdTe nanostructures," Angewandte Chemie - International Edition, vol. 45, no. 5, pp. 748-751, 2006.

[19] Z. Tang, Y. Wang, S. Shanbhag, and N. A. Kotov, "Spontaneous $\mathrm{CdTe} \rightarrow$ alloy $\rightarrow$ CdS transition of stabilizer-depleted CdTe nanoparticles induced by EDTA," Journal of the American Chemical Society, vol. 128, no. 21, pp. 7036-7042, 2006.

[20] Z. Tang, Y. Wang, S. Shanbhag, M. Giersig, and N. A. Kotov, "Spontaneous transformation of CdTe nanoparticles into angled Te nanocrystals: from particles and rods to checkmarks, X-marks, and other unusual shapes," Journal of the American Chemical Society, vol. 128, no. 20, pp. 6730-6736, 2006.

[21] H. Qian, E. Zhu, S. Zheng et al., "One-pot synthesis of biocompatible Te@phenol formaldehyde resin core-shell nanowires with uniform size and unique fluorescent properties by a synergized soft-hard template process," Nanotechnology, vol. 21, no. 49, Article ID 495602, 2010.

[22] H. Zhang, Z. Zhou, B. Yang, and M. Gao, "The influence of carboxyl groups on the photoluminescence of mercaptocarboxylic acid-stabilized CdTe nanoparticles," Journal of Physical Chemistry B, vol. 107, no. 1, pp. 8-13, 2003.

[23] U. K. Gautam and C. N. R. Rao, "Controlled synthesis of crystalline tellurium nanorods, nanowires, nanobelts and related structures by a self-seeding solution process," Journal of Materials Chemistry, vol. 14, no. 16, pp. 2530-2535, 2004.

[24] J. Ge and Y. Li, "Selective atmospheric pressure chemical vapor deposition route to CdS arrays, nanowires, and nanocombs," Advanced Functional Materials, vol. 14, no. 2, pp. 157-162, 2004.

[25] X. G. Peng, "An essay on synthetic chemistry of colloidal nanocrystals," Nano Research, vol. 2, pp. 425-447, 2009.

[26] K. L. Linge, "Trace element determination by ICP-AES and ICP-MS: developments and applications reported during 2006 and 2007," Geostandards and Geoanalytical Research, vol. 32, no. 4, pp. 453-468, 2008.

[27] H. S. Qian, S. H. Yu, J. Y. Gong, L. B. Luo, and L. F. Fei, "High-quality luminescent tellurium nanowires of several nanometers in diameter and high aspect ratio synthesized by a poly (vinyl pyrrolidone)- assisted hydrothermal process," Langmuir, vol. 22, no. 8, pp. 3830-3835, 2006.

[28] J. M. Song, Y. Z. Lin, Y. J. Zhan, Y. C. Tian, G. Liu, and S. H. $\mathrm{Yu}$, "Superlong high-quality tellurium nanotubes: synthesis, characterization, and optical property," Crystal Growth and Design, vol. 8, no. 6, pp. 1902-1908, 2008.

[29] L. Zhang, C. Wang, and D. Wen, "Preparation by hydrothermal techniques in a tungstosilicate acid solution system and optical properties of tellurium nanotubes," European Journal of Inorganic Chemistry, no. 22, pp. 3291-3297, 2009.

[30] R. Ochoa-Landín, O. Vigil-Galan, Y. V. Vorobiev, and R. Ramírez-Bon, "Chemically-deposited Te layers improving the parameters of back contacts for CdTe solar cells," Solar Energy, vol. 83, no. 1, pp. 134-138, 2009.

[31] A. K. Arora, M. Rajalakshmi, T. R. Ravindran, and V. Sivasubramanian, "Raman spectroscopy of optical phonon confinement in nanostructured materials," Journal of Raman Spectroscopy, vol. 38, no. 6, pp. 604-617, 2007.

[32] C. O’Sullivan, R. D. Gunning, A. Sanyal et al., "Spontaneous room temperature elongation of $\mathrm{CdS}$ and $\mathrm{Ag}_{2} \mathrm{~S}$ nanorods via oriented attachment," Journal of the American Chemical Society, vol. 131, no. 34, pp. 12250-12257, 2009.

[33] A. L. Rogach, L. Katsikas, A. Kornowski, S. Dangsheng, A. Eychmüller, and H. Weller, "Synthesis and characterization of thiol-stabilized CdTe nenocrystals," Berichte der Bunsengesellschaft, vol. 100, no. 11, pp. 1772-1778, 1996.

[34] L. Manna, L. W. Wang, R. Cingolani, and A. P. Alivisatos, "First-principles modeling of unpassivated and surfactantpassivated bulk facets of wurtzite CdSe: a model system for studying the anisotropic growth of CdSe nanocrystals," Journal of Physical Chemistry B, vol. 109, no. 13, pp. 6183-6192, 2005.

[35] Z. A. Peng and X. Peng, "Nearly monodisperse and shapecontrolled CdSe nanocrystals via alternative routes: nucleation and growth," Journal of the American Chemical Society, vol. 124, no. 13, pp. 3343-3353, 2002.

[36] L. Manna, E. C. Scher, and A. P. Alivisatos, "Synthesis of soluble and processable rod-, arrow-, teardrop-, and tetrapodshaped CdSe nanocrystals," Journal of the American Chemical Society, vol. 122, no. 51, pp. 12700-12706, 2000.

[37] Y. S. Xia, T. D. Nguyen, M. Yang et al., "Self-assembly of self-limiting monodisperse supraparticles from polydisperse nanoparticles," Nature Nanotechnology, vol. 6, pp. 580-587, 2011. 

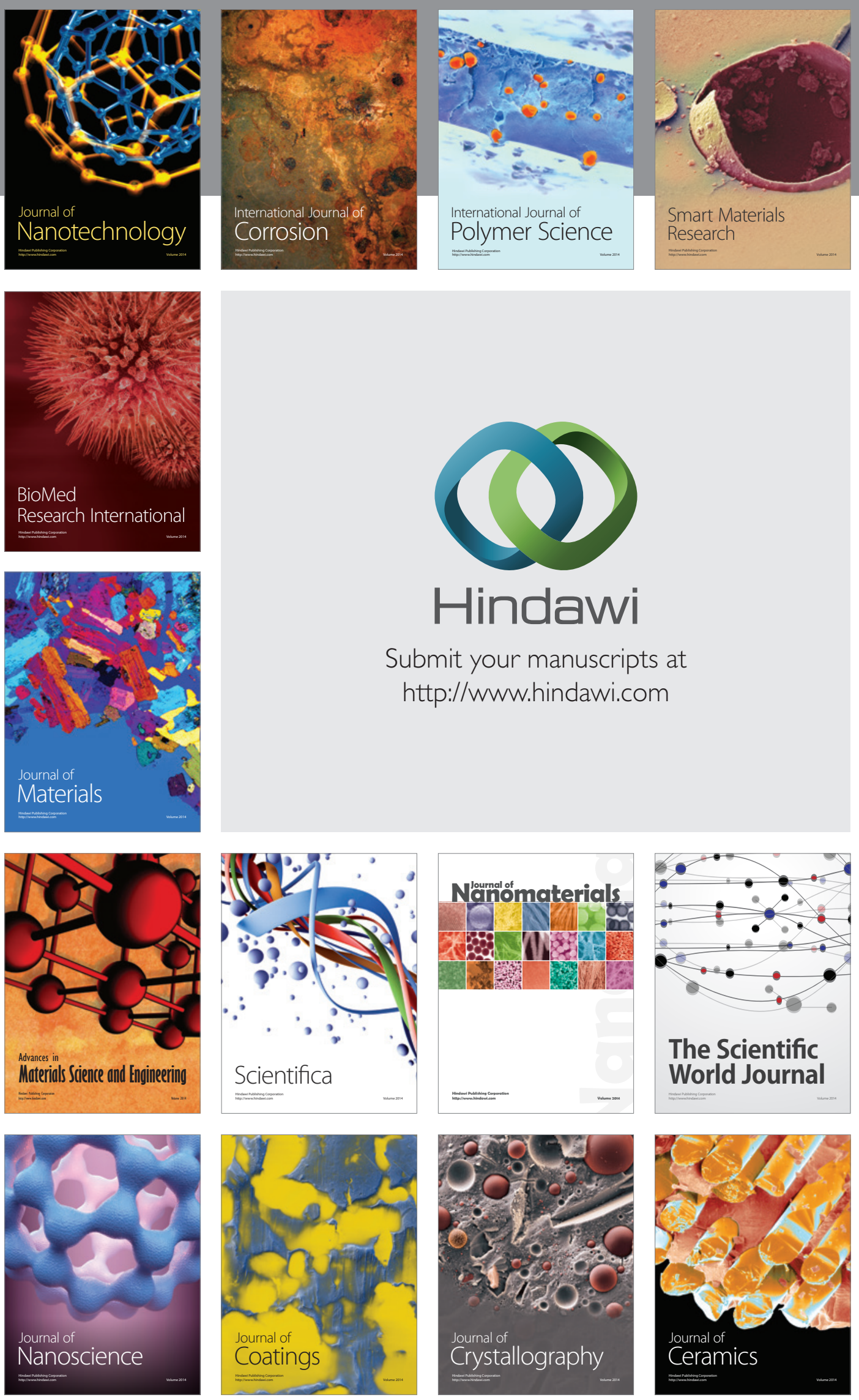

The Scientific World Journal

Submit your manuscripts at

http://www.hindawi.com

\section{World Journal}

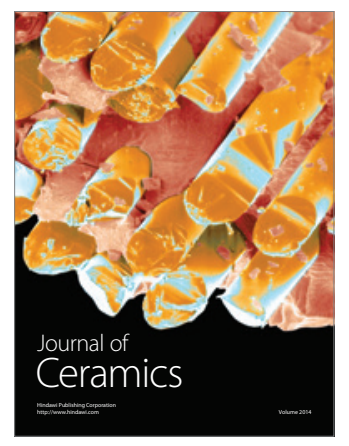

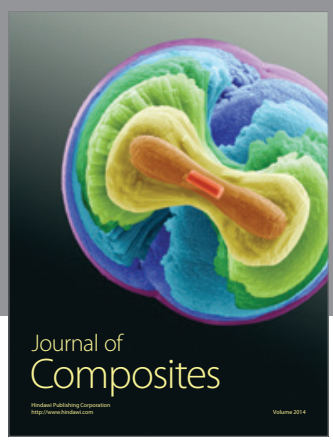
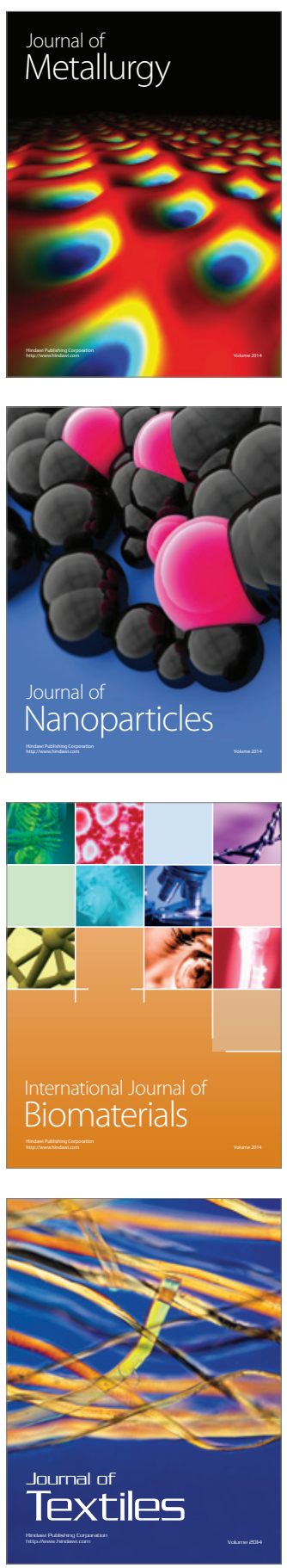